BANCA D'ITALIA

E U R O S I S T E M A

Questioni di Economia e Finanza

(Occasional Papers)

Risk-adjusted expectations of inflation

by Marco Casiraghi and Marcello Miccoli 

13 BANCA D'ITALIA

E U ROS I S T E M A

\section{Questioni di Economia e Finanza}

(Occasional papers)

Risk-adjusted expectations of inflation

by Marco Casiraghi and Marcello Miccoli

Number 286 - July 2015 
The series Occasional Papers presents studies and documents on issues pertaining to the institutional tasks of the Bank of Italy and the Eurosystem. The Occasional Papers appear alongside the Working Papers series which are specifically aimed at providing original contributions to economic research.

The Occasional Papers include studies conducted within the Bank of Italy, sometimes in cooperation with the Eurosystem or other institutions. The views expressed in the studies are those of the authors and do not involve the responsibility of the institutions to which they belong.

The series is available online at www.bancaditalia.it.

ISSN $1972-6627$ (print)

ISSN 1972-6643 (online)

Printed by the Printing and Publishing Division of the Bank of Italy 


\title{
RISK-ADJUSTED EXPECTATIONS OF INFLATION
}

\author{
By Marco Casiraghi* and Marcello Miccoli*
}

\begin{abstract}
We propose a new way to compute market-based risk-adjusted measures of inflation expectations. Borrowing from the finance literature, we study the ex-post excess return on inflation swap contracts - the difference between the swap rate at a given maturity and the realized inflation rate over the same horizon - which is an unbiased proxy of risk premia under the rational expectations hypothesis. The empirical results show that the risk premia on inflation swap rates at short-to-medium maturities can be predicted by macroeconomic variables that are present in agents' information set at the time the contract is signed, and that they vary counter-cyclically. This econometric analysis is then used to construct a measure of risk-adjusted inflation expectations so as to assess the role of risk premia in determining inflation swap rates. On this basis we find that the observed decline in inflation swap rates at short-to-medium maturities in 2014 was driven mainly by changes in inflation expectations.
\end{abstract}

Classificazione JEL: E52, G13.

Keywords: monetary policy, inflation swap, inflation expectations, risk premia.

\section{Contents}

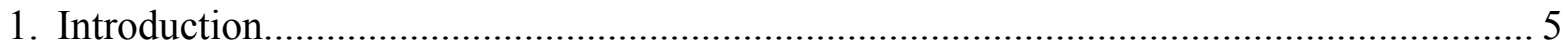

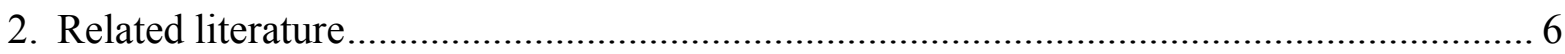

3. Econometric Approach ...................................................................................... 7

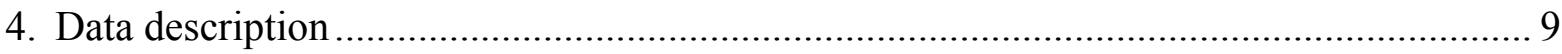

5. Empirical estimates and in-sample fit............................................................... 11

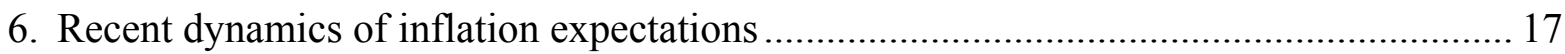

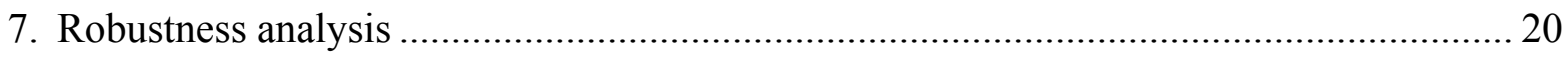

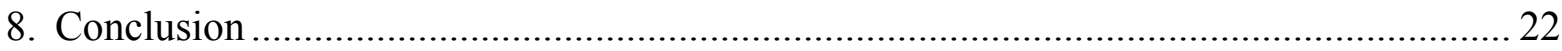

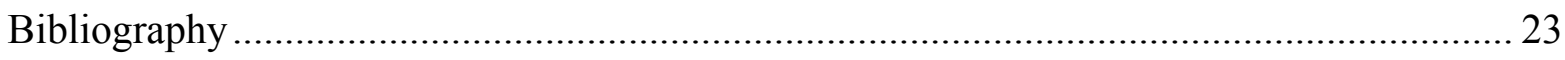

* Bank of Italy, Directorate General for Economics, Statistics and Research. E-mail: marco.casiraghi@bancaditalia.it, marcello.miccoli@bancaditalia.it. The views expressed here are those of the authors and do not necessarily represent in any way the views of the Bank of Italy. 



\section{Introduction}

Inflation expectations are probably the most often consulted figures in central banks. Longer-term expectations provide central bankers with a measure of their credibility, while expectations at shorter horizons reflect the effectiveness of monetary policy and the efficacy of their decisions. There are two sources of data on inflation expectations. First, there are surveys, which typically consist of periodic polls of professional forecasters, firms or individuals. Second, it is possible to obtain market-based measures by analyzing assets whose prices are linked in some way to inflation. Both sources have distinct advantages and drawbacks. Market-based expectations are available at high frequency, embody up-to-date information, and aggregate the expectations of a large pool of agents in the economy. At the same time they are imperfect measures of inflation expectations, since they are based on traded assets' prices that are affected by risk premia. Survey based expectations are not biased by these premia; however, their low frequency makes them less useful for real-time decision making. Moreover, it seems reasonable to assume that agents tend to form their expectations more carefully when their money is at stake. Clearly, given that both sources have distinct advantages, the attitude in policymaking has been to use both of them. Still the problem of how to filter out premia from market-based inflation expectation is on the table.

Available estimates of inflation risk premia differ among them in terms of sign, magnitude and dynamics, and it is arguably difficult to clearly identify the source of this heterogeneity in empirical results. ${ }^{1}$ In this work we propose a novel way to create market based risk-adjusted measures of inflation expectations. We focus on a widely used market instrument, namely inflation swaps contracts. Borrowing from the finance literature (Piazzesi-Swanson (2008), Cochrane-Piazzesi (2005)), we study the ex-post excess return - the difference between the inflation swap rate at a given maturity and the realized inflation rate over the same horizon - which is an unbiased proxy of the inflation risk premia under the rational expectations hypothesis.

Our empirical analysis documents that the risk premia on inflation swap rates at short-medium maturities can be predicted by macroeconomic variables in the information set of agents at the time of the inflation swap contract, and that they vary counter-cyclically. We then use the outcome of the econometric analysis to construct a measure of risk-adjusted inflation expectations in order to assess the role played by risk premia in determining the patterns of inflation swap rates observed in recent times.

Focusing on 2014, the results show that risk premia have been positive, although weakly decreasing over the year; risk-adjusted inflation expectations decreased in the second half of the year, after slightly increasing in the previous months. All in all, this suggests that the observed decline in market-based inflation expectations was driven mainly by changes in underlying expected inflation rather than by variation in risk

\footnotetext{
${ }^{1}$ Pericoli (2012) provides a comparison of some estimates of inflation risk premia found in the literature and shows that indeed there are stark differences among them.
} 
premia. The increase in inflation swap rate observed in the initial months of 2015 is then shown to be more likely linked to increase in expectation of inflation, probably due to the monetary policy measures adopted by the European Central Bank, rather than to changes in risk premia.

The paper is organized as follows. The following section presents the related literature; section 3 then outlines our identification strategy. Section 4 describes the data used, section 5 presents the results of the estimates and constructs the riskadjusted measures for inflation expectations; section 6 analyses the dynamics of the risk-adjusted measures in the period 2012-2015; section 7 describes some robustness analysis. Section 8 concludes.

\section{Related Literature}

There are two strands of literature related to this work. The first one is the line of works that documents the failure of the expectation hypothesis, and in particular the works of Cochrane and Piazzesi (2005) and Piazzesi and Swanson (2008), from which we borrow the methodology of the analysis of excess returns: both show how excess returns in bonds (Cochrane and Piazzesi (2005)) and in futures on federal funds rate (Piazzesi and Swanson (2008)) can be forecasted by appropriately chosen macroeconomic or financial variables.

The second strand of literature is related to the estimate of the inflation risk premia from market based expectation by using macroeconomic modelling techniques. Here the literature mostly focused on inflation expectations derived from inflation linked bonds, for larger data availability. Recent works in this field are Hördahl and Tristani (2014), and Hördahl and Tristani (2012) in which they use a model of macroeconomic and term structure dynamics to estimate the size and dynamics of the inflation risk premia in the euro-area; Fleckenstein, Longstaff and Lustig (2013) that uses both inflation swaps and inflation options in order to estimate the inflation risk premia for the US economy; Haubrich, Pennacchi and Richken (2012) in which a factor model is developed and applied to the US inflation swaps data; Andreasen (2012) that estimates a DSGE model and derives implication for the size and evolution on the inflation risk

premia in the UK economy. Compared with these works, our identification strategy has the advantage on relying only one assumption, that of rational expectations; however, the arbitrary choice of regression variables might be a drawback of our approach. Unfortunately, the different time sample and object of study of these papers implies that we cannot compare their estimates with ours. 


\section{Econometric approach}

A zero-coupon inflation swap is a contract whereby two counterparties agree to exchange at some future date a predetermined amount against a variable amount linked to the realized inflation rate over the maturity of the contract. The leg depending on accrued inflation (referred to as the inflation, or the floating, leg) will vary solely on the basis of the final price index reference value between the beginning and end of the period of the swap. The cash flow on the fixed leg is predetermined by the quoted swap rate and is effectively a break-even inflation rate; both parties will break even on the trade (i.e., the net cash flow at maturity will be nil) only if annualized average inflation over the swap's period is equal to the initial fixed rate. As such, the fixed rate, agreed at the trade date, should reflect expected future inflation over the time of the contract.

However, uncertainty and risk aversion imply that the inflation swap rate includes an additional term, namely inflation risk premia. Although inflation swap rates may also include premia associated with liquidity and counterparty risks, our analysis will abstract from considering premia other than those owing to inflation. The reason is that counterparty risks seem to be limited, since the swap contract involves only the exchange of the net settlement at maturity and never of the notional amount of the contract. Regarding liquidity premia, even if most evidence suggests that inflation swap markets are sufficiently deep and liquid, we include the bid-ask spread on swap contracts among the covariates in order to control for potential changes in the correspondent market liquidity.

By letting $r_{t}^{(n)}$ be the inflation swap rate traded at time $t$ with maturity $n$, we can then write such rate as

$$
r_{t}^{(n)}=E_{t} \pi_{t, t+n}+I R P_{t}^{(n)}
$$

where $E_{t} \pi_{t, t+n}$ is the expected inflation rate over the maturity of the contract. The presence of the inflation risk premia, $I R P_{t}^{(n)}$, which can be positive or negative and varying over time, is the fundamental problem in interpreting inflation swap rates as measures of expected future inflation.

There is a large literature that has tried to estimate inflation risk premia: unfortunately one of the main takeaways of the literature is that there is large uncertainty on the estimates, which seem to be model dependent. Essentially the core of the issue is that since one can only observe the sum of the terms on the right hand side of equation (1), identification of the expectation term is strictly conditional on the modelling assumptions taken. Moreover, in the existing literature it is not always clear how different assumptions beget different estimates.

Differently from previous work, in order to tackle this identification problem we will take a side approach, borrowing from the finance literature. First, we are going to posit that the inflation risk premia can be described by a regression model where regressors 
are time $t$ economic variables, $X_{t}$ (we will discuss which regressors to use later in the paragraph):

$$
I R P_{t}^{(n)}=\beta^{\prime} X_{t}+\tilde{\varepsilon}_{t} .
$$

Second, since the inflation risk premia is not directly observable, we will focus on the difference between the inflation swap rate and the realized inflation rate, which, using common terminology, is defined as the ex-post "excess return":

$$
e r_{t+n}^{(n)} \equiv r_{t}^{(n)}-\pi_{t, t+n}
$$

Using equation (1), we can rewrite the excess return as:

$$
e r_{t+n}^{(n)}=I R P_{t}^{(n)}+\left(E_{t} \pi_{t, t+n}-\pi_{t, t+n}\right),
$$

where the term in parentheses is the forecast error on inflation. Under rational expectations the forecast error is uncorrelated with the realization of other economic state variables at time $t$. This assumption allows us to use excess return as a proxy of the inflation risk premia in the regression model. To show this, one can add the forecast error term to both sides of the regression model in equation (2) to obtain:

$$
I R P_{t}^{(n)}+\left(E_{t} \pi_{t, t+n}-\pi_{t, t+n}\right)=\beta^{\prime} X_{t}+\tilde{\varepsilon}_{t}+\left(E_{t} \pi_{t, t+n}-\pi_{t, t+n}\right),
$$

and combining it with equation (4), we get

$$
e r_{t+n}^{(n)}=\beta^{\prime} X_{t}+\varepsilon_{t+n}
$$

with the error term, $\varepsilon_{t+n}$, including the error forecast term. The rational expectations assumption allows us to say that the error term $\varepsilon_{t+n}$ is orthogonal to the regressors $X_{t}$. Therefore, we will be able to obtain unbiased estimates of the coefficients $\beta$ by estimating the regression model in equation (6), which thus represents our main econometric specification. The fitted values of our regression model will be the estimate of the inflation risk premia, since the assumptions associated with OLS estimation imply that

$$
E\left[\operatorname{er}_{t+n}^{(n)} \mid X_{t}\right]=\hat{\beta}^{\prime} X_{t}
$$

where $E\left[\cdot \mid X_{t}\right]$ is the expectation operator conditional on the information set $X_{t}$ and $\hat{\beta}$ are the estimated values of the coefficients. Using equation (4) and the rational expectations assumption, the left hand side becomes:

$$
E\left[\mathrm{er}_{\mathrm{t}+\mathrm{n}}^{(\mathrm{n})} \mid \mathrm{X}_{\mathrm{t}}\right]=\mathrm{E}\left[\operatorname{IRP}_{\mathrm{t}}^{(\mathrm{n})}+\left(\mathrm{E}_{\mathrm{t}} \pi_{\mathrm{t}, \mathrm{t}+\mathrm{n}}-\pi_{\mathrm{t}, \mathrm{t}+\mathrm{n}}\right) \mid \mathrm{X}_{\mathrm{t}}\right]=\mathrm{E}\left[\operatorname{IRP}_{\mathrm{t}}^{(\mathrm{n})} \mid \mathrm{X}_{\mathrm{t}}\right]
$$

Our identification assumption rests therefore on the rational expectation hypothesis. Although it is hard to argue that such hypothesis never fails, it is more likely to hold for short and medium term horizons analysis, i.e. for short and medium-term inflation swap 
rate. The reason is that the impact of potential learning processes, which would invalidate our assumption, is small over those horizons.

With this caveat in mind, what does the inflation risk premia depend on, what are the variables to be included as explanatory variables? Clearly one has to formulate a model in order to answer this question, and even after doing so, it will depend on some unobservable state variables. We will take a model-less approach, trying to be as simple and as parsimonious as possible, even though we share the concern with the reader that the choice of explanatory variables in a model-less approach is arbitrary.

In order to limit this issue we will be guided partly by previous literature. Piazzesi and Swanson (2008) show how excess returns on the federal funds rate can be well predicted by some indicator of the economic cycle. Thus we will include as regressors the unemployment rate and the manufacturing PMI index in the euro area. We will also include the expected monetary policy stance, as proxied by overnight index swap rates as one factor that can influence macroeconomic variables in the near future.

The advantage of focusing on the excess return also is that this can be easily used to create risk-adjusted forecast of inflation from the inflation swap rates. Re-arranging equation (1) and using the estimates of our regression model, risk-adjusted inflation expectations thus obtain:

$$
E_{t} \widehat{\pi_{t, t}+n}=r_{t}^{(n)}-\hat{\beta}^{\prime} X_{t} .
$$

To the extent that the inflation risk premia is forecastable (so that regression coefficients are significant), then by using the inflation swap rates and the fitted values of our regression, equation (7) gives a risk-adjusted measure of expectations of inflation.

\section{Data description}

In our analysis we solely focus on inflation swaps for the euro area. These contracts are indexed to the non-seasonally adjusted Euro HICPXT (Harmonised Index of Consumer Prices excluding Tobacco), as reported by Eurostat in its first flash estimate, which means it is not affected by future revisions. ${ }^{2}$ The inflation indexation mechanism in a swap is subject to a three-month lag. This means a euro inflation swap traded on any given day of a particular month has the same starting index reference value, which is the published index value three months before. For example, all standard euro HICPxT swaps traded during January 2015 pay inflation accruing from October 2014, which is referred to as the base month. In February 2015, the base month for all standard euro area inflation swaps shifts one month and changes to November 2014 and so forth. We take inflation swaps rate at different maturities as provided by Bloomberg and we consider the monthly average of inflation swaps rates through each month. In order to

\footnotetext{
${ }^{2}$ This index is available as OATEI01 on Reuters and as CPTFEMU on Bloomberg.
} 
address the issue of overlapping observations we will control for potential serial correlation in the residuals, up to 50 lags. As we said earlier, we also compute the bidask spread for each maturity, which can be interpreted as a measure of volatility and thus illiquidity in the relevant markets.

Eurostat also publishes several monthly unemployment series that are calculated first at the level of each Member State, and then aggregated at the euro area level. Throughout the analysis, we will use the seasonally adjusted unemployment rate, which is defined as the number of people unemployed as a percentage of the labour force.

The PMI (Purchasing Managers' Index) series are monthly surveys of selected companies compiled by Markit. Questionnaire responses come from panels of senior purchasing executives (or similar) at a large number of companies, and thus they provide advance insight into the health of private sector economy. In our regressions, we use the manufacturing PMI index, that is a composite index based on a weighted combination of the following five survey variables (weights shown in brackets): new orders (0.3), output (0.25), employment (0.2), suppliers' delivery times (0.15), and stocks of materials purchased (0.1). The overall PMI index for manufacturing firms can be thought as a snapshot of the conditions this specific economic sector expects to face at the time of the survey. In details, an index reading of over 50.0 indicates an improvement, while anything below 50.0 suggests a deterioration. The further away from 50.0 the PMI index is, the stronger the change over the month.

Finally, 3-month overnight index swap (OIS) rates are included among the explanatory variables in order to control for expectations on future monetary stance. 3month OIS are indeed swap contracts, whose variable leg is pegged to the average EONIA observed over the following three months.

Our sample consists of monthly observations from June 2004 to April 2015. Unfortunately, it is not possible to move backward the starting date due to the lack of observations on inflation swaps in the euro area, which were not traded before June 2004. ${ }^{3}$ As specified earlier, we take the monthly average of daily variables (inflation swap prices and OIS rates) in order to standardize the frequency of the database and also in order to limit the impact of volatility that would otherwise be amplified if considering daily data (e.g. the last day of each month). ${ }^{4}$

Table 1 reports the summary statistics of the main variables. Throughout the analysis, $e r_{t}^{(n)}$ stands for the excess return of the inflation swap rate, computed as the difference between the inflation swap rate of $n$-year maturity traded in month $t-12 * n$ and the realized $n$-year inflation rate in month $t$ (all figures are shown in annualized values). Given that the initial sample date is fixed (the month inflation swap were first

\footnotetext{
${ }^{3}$ The initial date at which inflation swap contract were first traded differs among maturities.

${ }^{4}$ As a robustness check we have redone all estimates using last day of month data, and results are similar.
} 
traded), the number of observations we can use to compute the excess return decreases with the maturity of the inflation swap rate.

Table 1: summary statistics

\begin{tabular}{|c|c|c|c|c|c|}
\hline VARIABLES & $\mathrm{N}$ & mean & sd & $\min$ & $\max$ \\
\hline$e r_{t}^{(1)}$ & 122 & -0.026 & 0.888 & -2.036 & 2.391 \\
\hline$e r_{t}^{(2)}$ & 115 & 0.048 & 0.713 & -1.319 & 1.950 \\
\hline$e r_{t}^{(3)}$ & 98 & 0.105 & 0.572 & -0.833 & 1.276 \\
\hline$e r_{t}^{(4)}$ & 86 & 0.172 & 0.403 & -0.629 & 1.175 \\
\hline$e r_{t}^{(5)}$ & 76 & 0.293 & 0.224 & -0.360 & 1.068 \\
\hline Pmi & 131 & 50.8 & 5.2 & 33.5 & 59 \\
\hline $\begin{array}{l}\text { Unemployment } \\
\text { rate }\end{array}$ & 130 & 9.7 & 1.5 & 7.2 & 12.1 \\
\hline 3 month OIS & 131 & 1.5 & 1.5 & -0.1 & 4.3 \\
\hline $\begin{array}{c}\text { Bid-ask spread } \\
\text { 1y swap }\end{array}$ & 131 & 0.132 & 0.104 & 0.026 & 0.873 \\
\hline $\begin{array}{l}\text { Bid-ask spread } \\
2 y \text { swap }\end{array}$ & 131 & 0.095 & 0.053 & 0.033 & 0.297 \\
\hline $\begin{array}{c}\text { Bid-ask spread } \\
\text { 3y swap }\end{array}$ & 131 & 0.082 & 0.051 & 0.033 & 0.279 \\
\hline $\begin{array}{c}\text { Bid-ask spread } \\
4 y \text { swap }\end{array}$ & 131 & 0.060 & 0.023 & 0.032 & 0.150 \\
\hline $\begin{array}{c}\text { Bid-ask spread } \\
5 y \text { swap }\end{array}$ & 131 & 0.050 & 0.020 & 0.029 & 0.153 \\
\hline
\end{tabular}

\section{Empirical estimates and in-sample fit}

We first investigate whether average excess returns on inflation swap rate are zero on average in the sample period, so we run the regression:

$$
e r_{t}^{(n)}=\alpha^{(n)}+\varepsilon_{t}^{(n)}
$$

for different horizons $n$. Table 2 shows results from regression (8) for the forecast horizons $n=1, \ldots, 5$ years over the entire period for which we have data on inflation swaps. We compute standard errors using the heteroskedasticity and autocorrelationconsistent procedure, allowing for excess returns to be serially correlated due to contract overlap. Therefore, we report HAC standard errors throughout the entire analysis. ${ }^{5}$ Moreover, to control for small-sample distributional properties, we compute small-sample statistics ( $F$ and t-statistics) instead of large-sample statistics (chi-squared and z-statistics).

\footnotetext{
${ }^{5}$ We rely on the Bartlett kernel-based estimator, with bandwidth parameter set to one in baseline estimates. In the robustness section we check the sensitivity of the variance-covariance matrix estimation to different bandwidth parameters.
} 
Table 2: equation (8) estimates

\begin{tabular}{llllll}
\hline \multicolumn{1}{c}{$e r_{t}^{(1)}$} & \multicolumn{1}{c}{$e r_{t}^{(2)}$} & \multicolumn{1}{c}{$e r_{t}^{(3)}$} & \multicolumn{1}{c}{$e r_{t}^{(4)}$} & \multicolumn{1}{c}{$e r_{t}^{(5)}$} \\
\hline \multirow{2}{*}{ Constant } & & & & & \\
& -0.0264 & 0.0478 & 0.105 & $0.172^{* * *}$ & $0.293^{* * *}$ \\
& $(0.112)$ & $(0.0925)$ & $(0.0803)$ & $(0.0594)$ & $(0.0339)$ \\
Observations & 122 & 115 & 98 & 86 & 76
\end{tabular}

HAC standard errors in parentheses. ${ }^{* * *} \mathrm{p}<0.01,{ }^{* *} \mathrm{p}<0.05,{ }^{*} \mathrm{p}<0.1$, significance values based on small-sample statistics. Rates are at annualized value, percentage points. Sample: 2004:6-2015:4

As can be seen in Table 2, average excess returns is not significantly different from zero on the 1, 2 and 3 years maturity, while it is significantly positive for longer horizons. Even though the excess return is zero on overage for shorter maturities, there is large variability over time. Figure 1 plots the realized excess return on the 1-year inflation swap rate from October 2005 to April 2015. The time variation in this realized excess return series has been large, ranging from -204 to 239 basis points (at annualized rates).

\section{Figure 1}

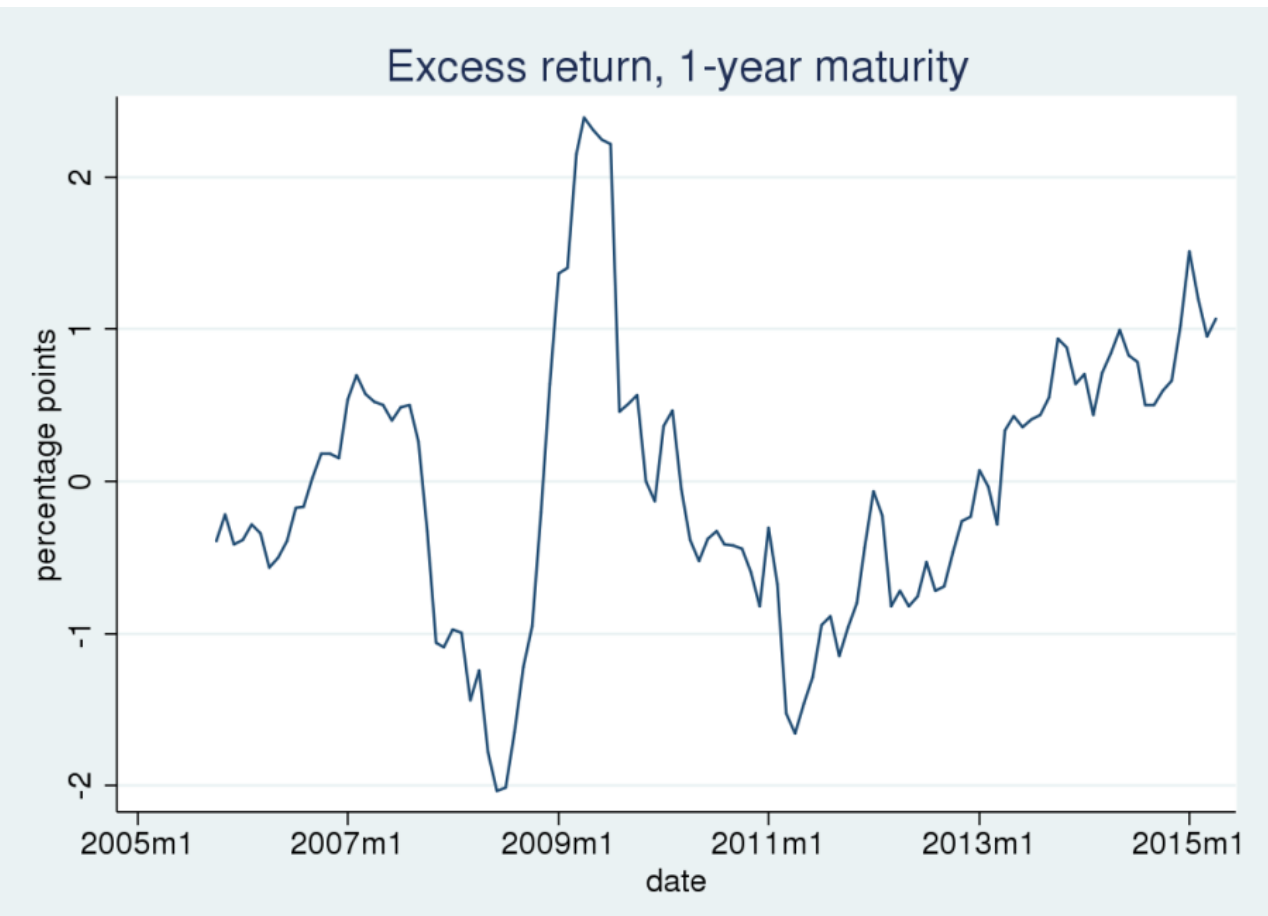


Given that excess returns have showed large variations over time, it is crucial to investigate whether they represent only forecast errors or also risk premia, and whethere the latter can be predicted by macroeconomic or financial business-cycle indicator. In order to do so, we turn to our main analysis running regressions of the form:

$$
e r_{t}^{(n)}=\alpha^{(n)}+\beta^{(n)^{\prime}} X_{t-12 * n+3}+\varepsilon_{t}^{(n)}
$$

where $X_{t}$ is a vector of variables known to financial markets in month $t-12 * n+3,{ }^{6}$ that is when the inflation swap is traded. ${ }^{7}$ As we specified in section 3 , we use as regressors closely related indicator of real activity that are available at monthly frequency: the PMI index value, the unemployment rate in the euro area, and the 3-months OIS rate (average over the month). ${ }^{8}$

Table 3 reports the results from regression equation (9). Except for the that on the bidask spread, all coefficients are significant when maturities up to 3 years are considered, and with the same estimated sign across all maturities. The magnitude of these coeffients varies slightly across maturities, though all within a close neighborhood. Signs are consistent with the evidence that risk premia are counter-cyclical (Piazzesi and Swanson (2008)), but for the coefficient on the OIS rate. However, since the OIS rate and the unemployment rate are highly correlated, the coefficient on the former only captures the variation orthogonal to the latter. Therefore one should not read the positive coefficient on the OIS rate as necessarily indicating a prociclicality of the risk premia.

\footnotetext{
${ }^{6}$ Note that the inflation swap has an indexation lag, as described in the previous section.

${ }^{7}$ Since inflation swaps data are averages over the month, we considered the information as available to the markets in month $t$ if it was released in the first part of the month. Also, notice that information available to markets in month $t$ does not usually refer to month $t$ data. For instance unemployment data for the euro area is usually released at the beginning of the month, but it refers to the unemployment rate two months before the data release; the lag in the case of the PMI index is only one month. We took care of these lags in the estimates. OIS rates enter the regression as same month average.

${ }^{8}$ For longer maturities, we have also run regression with 1-year or longer OIS rates. Results do not substantially differ.
} 
Table 3: equation (9) estimates, baseline

\begin{tabular}{lccccc}
\hline & $e r_{t}^{(1)}$ & $e r_{t}^{(2)}$ & $e r_{t}^{(3)}$ & $e r_{t}^{(4)}$ & $e r_{t}^{(5)}$ \\
\hline \multirow{2}{*}{ Pmi } & & & & & \\
& $\left(0.0845^{* * *}\right.$ & $-0.112^{* * *}$ & $-0.0559 * * *$ & 0.00566 & -0.0145 \\
Unemployment & $0.800^{* * *}$ & $(0.0166)$ & $(0.0146)$ & $(0.0179)$ & $(0.0163)$ \\
& $(0.104)$ & $\left(0.0976^{* * *}\right.$ & $0.788^{* * *}$ & 0.285 & 0.268 \\
OIS 3m & $0.781^{* * *}$ & $0.840^{* * *}$ & $(0.168)$ & $(0.245)$ & $(0.215)$ \\
& $(0.160)$ & $(0.0969)$ & $(0.116)$ & $(0.170)$ & $(0.159)$ \\
Bid-ask spread & $1.805^{* * *}$ & $-3.713^{* *}$ & -2.104 & -0.318 & -1.591 \\
& $(0.631)$ & $(1.533)$ & $(1.582)$ & $(2.339)$ & $(1.566)$ \\
Constant & $-4.905^{* * *}$ & -2.039 & $-5.511^{* * *}$ & $-3.310^{*}$ & -1.687 \\
& $(0.959)$ & $(1.719)$ & $(1.902)$ & $(1.983)$ & $(1.493)$ \\
& & & & & \\
Observations & 120 & 108 & 96 & 84 & 72 \\
R-squared & 0.360 & 0.682 & 0.512 & 0.380 & 0.121 \\
& & & & & \\
\hline
\end{tabular}

Overall, our regression model seems to explain quite well the inflation risk premia at short and medium term horizons, with $R^{2}$ values ranging from 0.32 at the 1 -year maturity to 0.66 at the 2-year maturity, while it does poorly when maturity exceeds the 3 year horizon. Several explanations can be put forward for this. As we already argued in section 3 , learning process on the data generating process of inflation are more likely to be present over long horizons, invalidating our estimation procedure. Moreover, the very long time length between the trade of the inflation swap and the actual inflation realization probably generates a lot of noise that blurs the cyclicality of the risk premia. Finally, the reduced number of observations available might also play a role.

Figure 2 plots the excess return at the 1 year maturity (as in figure 1) together with the estimate of the inflation and liquidity risk (the in-sample regression prediction). The estimated risk premia is roughly constant, few decimals around zero, for inflation swap traded up to the beginning of 2008. ${ }^{9}$ It then spikes up in the last month of 2008 (mostly due to the liquidity risk premia) before becoming negative for trades conducted in 2009, reaching a minimum value of -1.2 percent. It has since then increased, becoming positive at the end of 2011, standing at around 0.2 percent for the one-year inflation swap traded at the beginning of 2014.

\footnotetext{
${ }^{9}$ Note that the time on the $x$-axis refers to the time of the realized inflation rate. Since the plot refers to the one-year horizon inflation swap, one should read the estimated risk premia at time $t$ as the one prevailing on the one-year inflation swap contract traded roughly one year before $t$.
} 
Figure 2

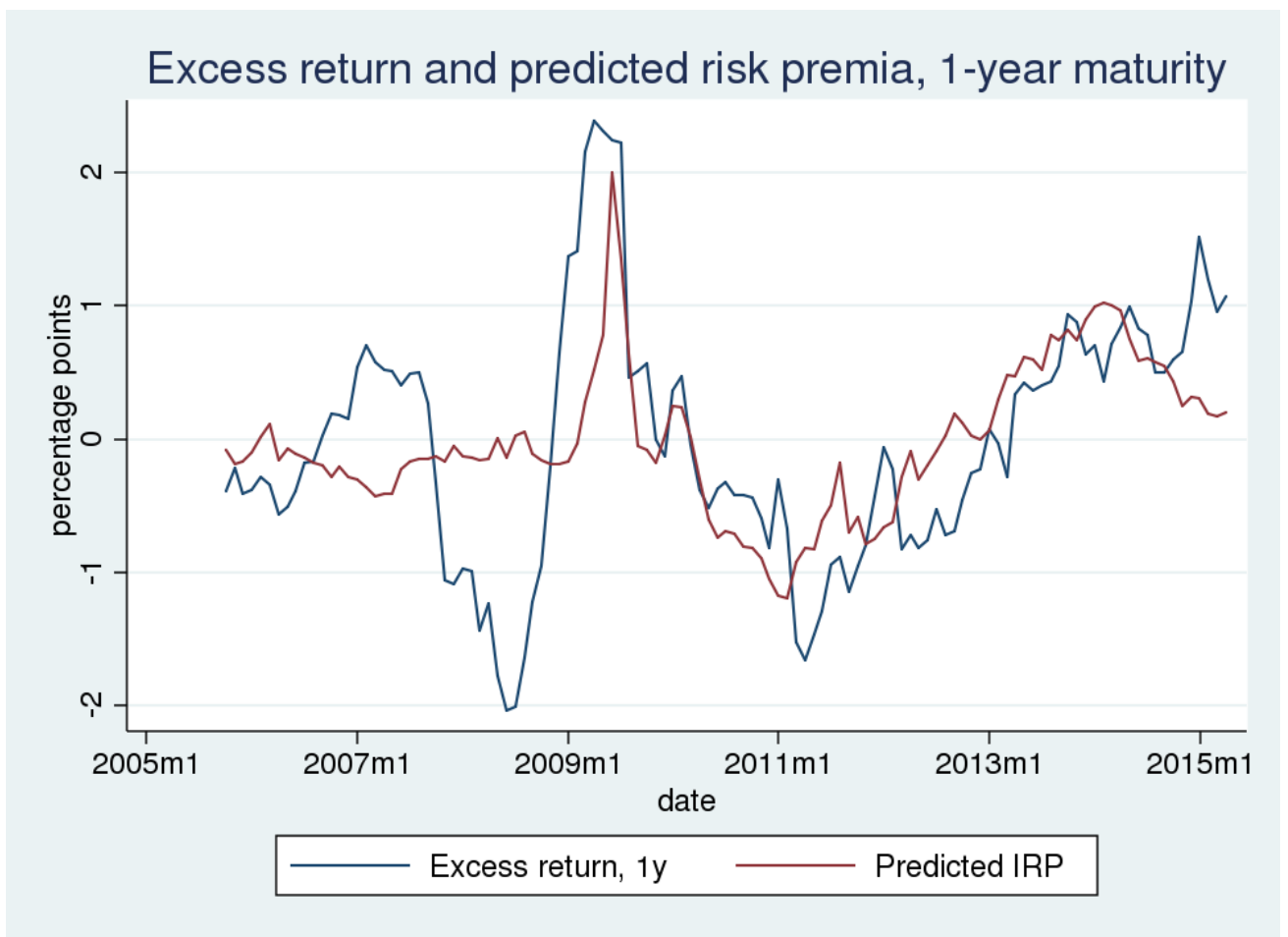

Given our regression model, we can backtrack what was the expected inflation rate predicted by the model over the sample. To do so, as in equation (7) we subtract the estimated risk premia from the inflation swap rates. Figure 3 shows the risk adjusted measure of expected inflation obtained using our regression model on the one-year excess return and the realized one-year inflation rate. While not a test of the goodness of the analysis, it is comforting to see that the regression model tracks closely realized inflation in in-sample estimates. 


\section{Figure 3}

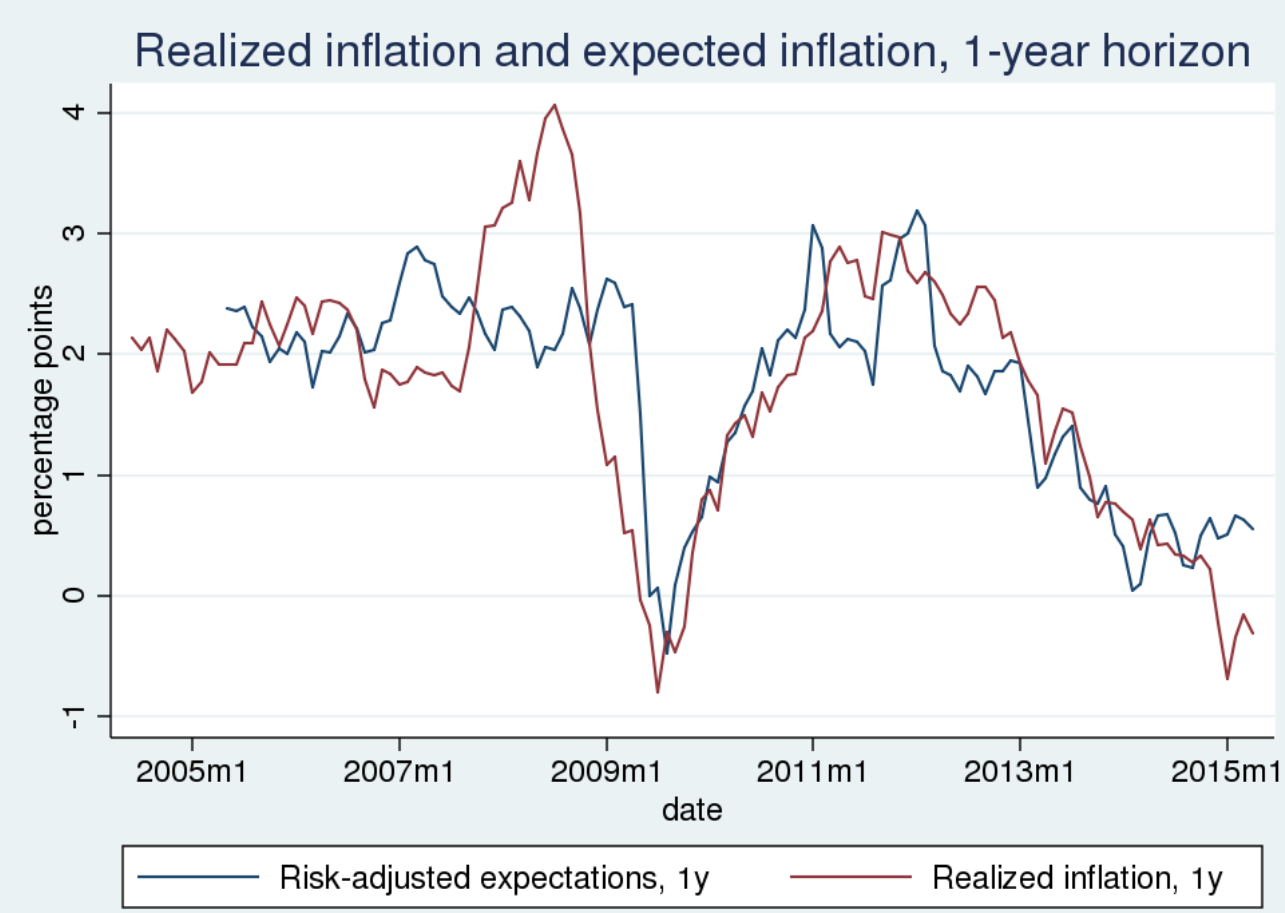

In our estimation strategy, the residuals of the regression correspond to the forecast error plus the error of the model estimating the inflation risk premia (see equation (6)). While clearly we cannot decompose the two, we can check whether the residuals of our regressions resemble the forecast error determined by survey based expectations of inflation. In order to do so, we compute the forecast error of inflation expectations three-quarter ahead, which are collected quarterly by Consensus for the euro area. ${ }^{10}$ Due to the indexation lag of inflation swap contract, these expectations refer to the same forecasting horizon as the one-year inflation swap.

Figure 4 plots the forecast error of the Consensus expectations together with the residuals of the regression equation when using the one-year maturity. The two series broadly match, with correlation coefficient between the two standing at 0.74 , thus pointing to further evidence that the regression model is able to capture the inflation risk premia.

\footnotetext{
${ }^{10}$ We constructed the forecast error as the difference between the Consensus expected inflation 3 quarters ahead and the realized HICP inflation rate.
} 
Figure 4

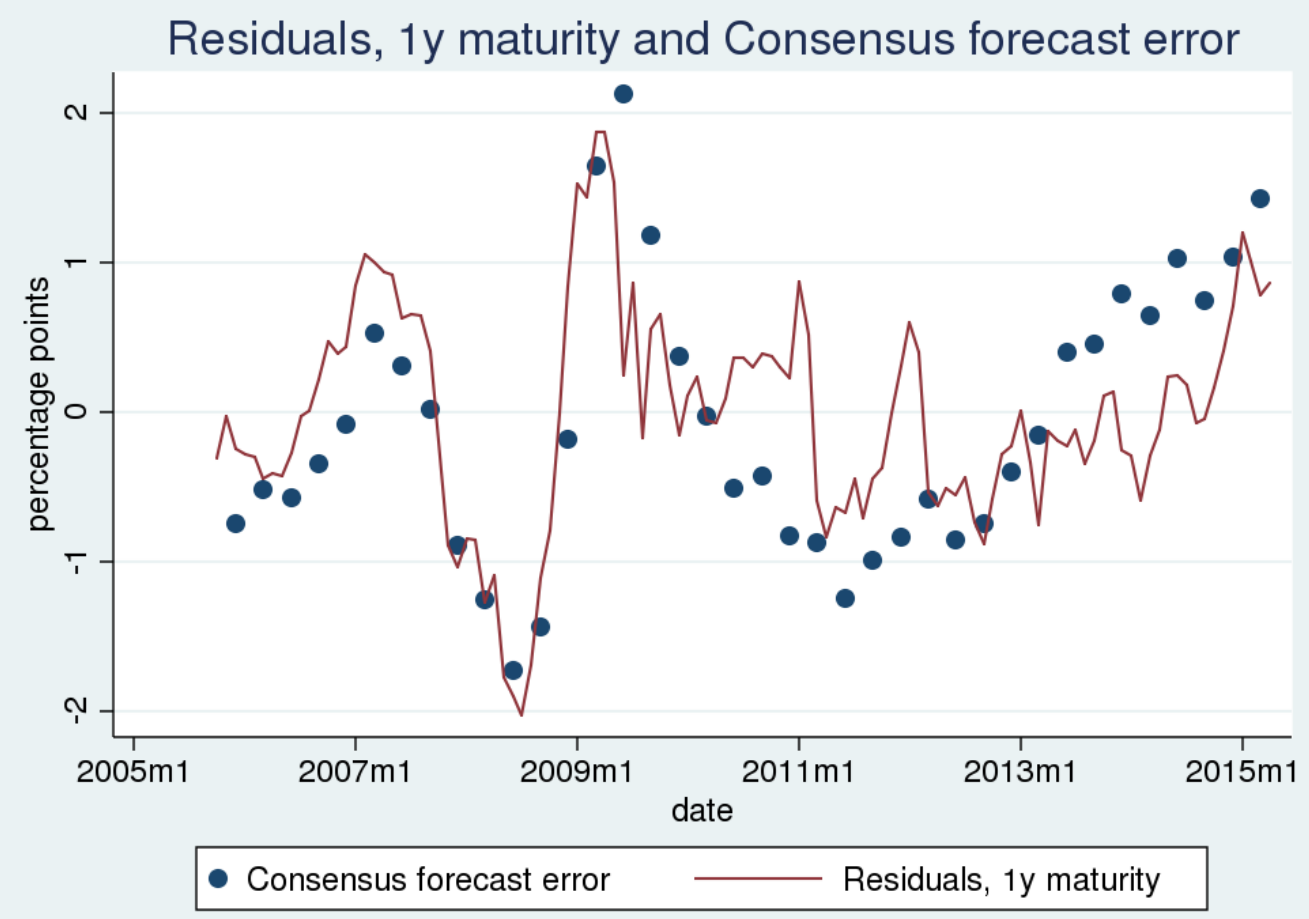

\section{Recent dynamics of inflation expectations}

We now turn to the key question, which is the motivation of our work: what do inflation swap rate tell us about inflation expectations once we correct for inflation risk premia? In particular, we are interested in investigating whether the decline in inflation swap rates since January 2012 - soon after the beginning of the European sovereign debt crisis - was driven by a fall in risk premia or in inflation expectations. Given the results of our analysis, we will focus on the horizons shorter than 3 years, since estimated coefficients are not significant when using longer maturities inflation swap rates. Therefore, our results are related to horizons that are of crucial importance for monetary policy.

We start with considering 1-year inflation expectations. Figure 5 shows the inflation swap rate on the 1-year maturity traded in the month indicated on the $x$-axis (red line) and our measure of risk-adjusted expectation of inflation when using our 1-year estimate (blue line). Given the short time horizon, it is not surprising that the riskadjusted expectations track well both the level and the development of the 1-year inflation swap. Nevertheless, the difference between the two measures increased significantly during 2012 and went back to almost zero towards the end of 2014. More 
important, Figure 5 shows that the decline in 1-year inflation swaps observed in 2014, and particularly in the second half of the year, was not driven by a fall in risk premia. ${ }^{11}$

\section{Figure 5}

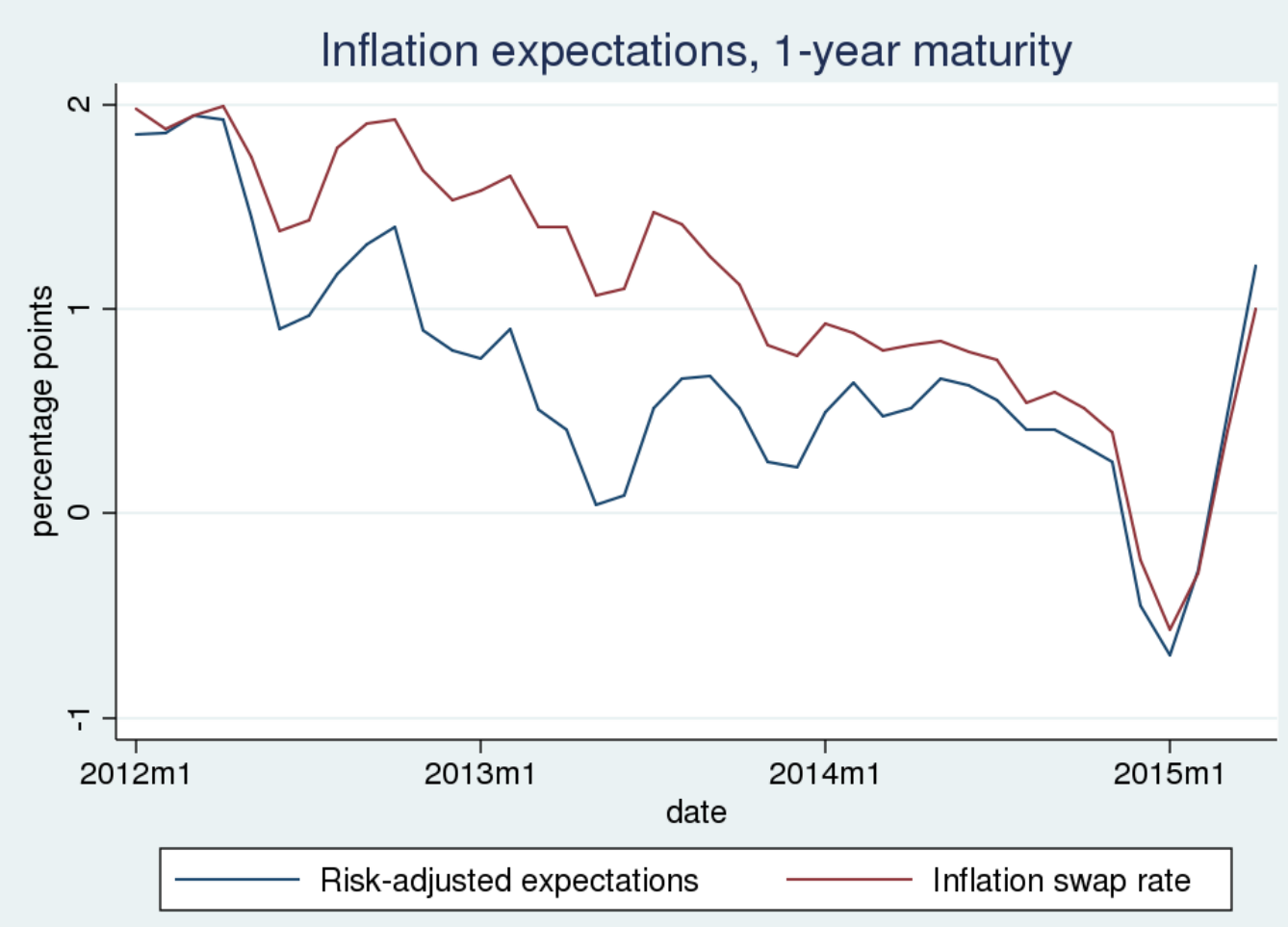

When moving to horizons beyond one year, in order to give easily interpretable measures of inflation, we compute 1-year forward rates. In particular, figures 6 and 7 report the 1-year ahead and the 2-year ahead forward rate, respectively, as implied by our risk-adjusted measures of inflation (blue line) and by swap contracts (red line).

The empirical evidence on inflation forward rates confirms that risk premia have been positive from the beginning of 2012 to the end of 2014. However it must be noted that the level of the risk-adjusted expectation of inflation on the 2-year ahead forward rate is very low. Notwithstanding this drawback of our estimation procedure, it is informative to analyze the dynamics of the risk-adjusted measure of expectations of inflation. Both the 2-year ahead and 3-year ahead forward rates seem to increase in the first half of 2014, once controlling for risk premia. This is line with the positive sentiment about economic prospects for the euro area at that time. Inflation expectation started declining again in the second semester of 2014, after agents realized that economic

\footnotetext{
${ }^{11}$ For further evidence on the decline of inflation of inflation expectations in the euro area during 2014, see Cecchetti, Natoli and Sigalotti (2015).
} 
recovery was going to be more modest than expected. In this case, the pattern is common to both inflation swaps and risk-adjusted measures.

Overall, risk-adjusted measures of expected inflation suggest that the dynamics of inflation swap rates in 2014 were not the result of swings in premia, but more likely reflected changes in in underlying expectations of market operators with respect to future inflation in the euro area.

\section{Figure 6}

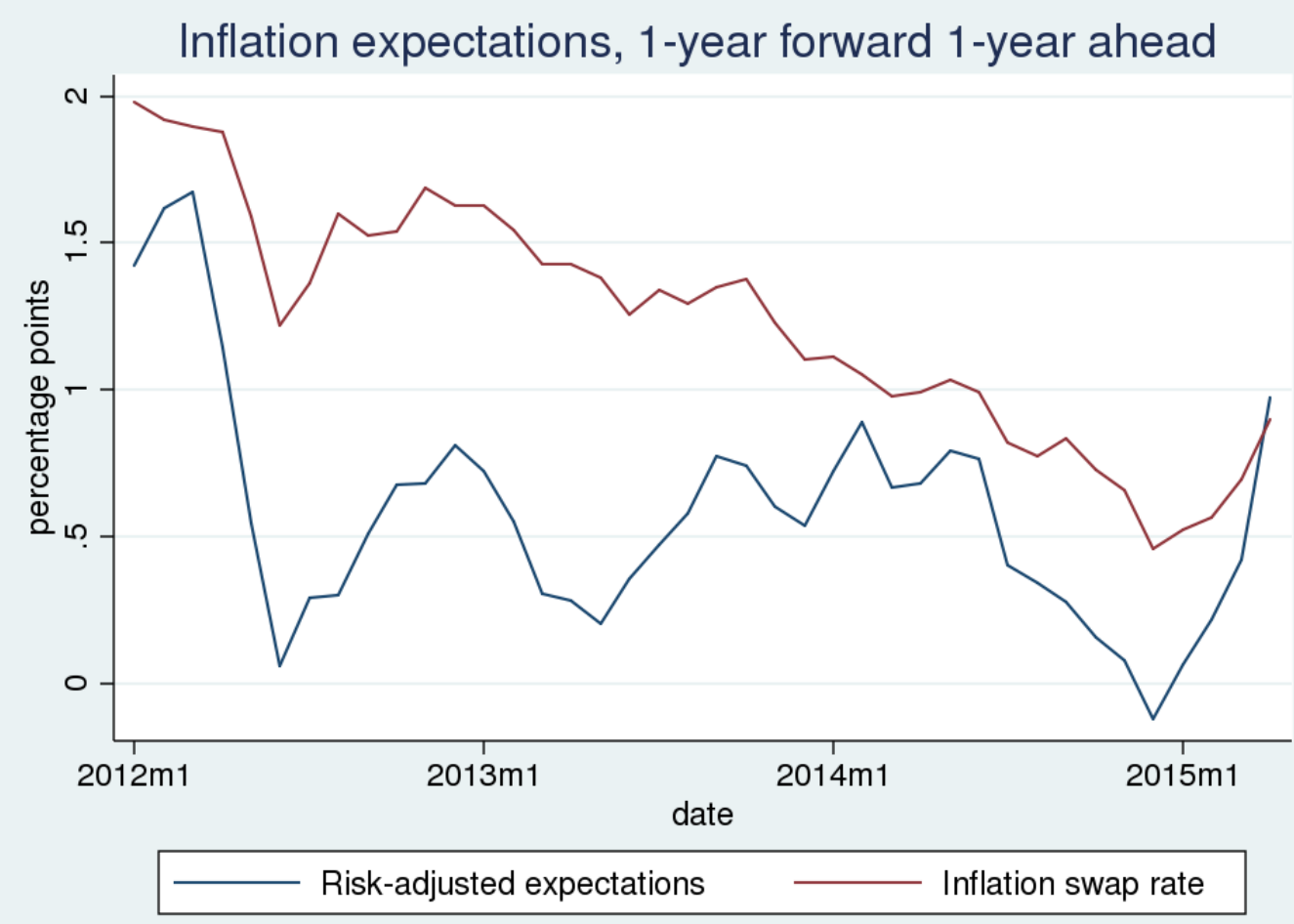

Similarly, the increase observed in the first month of 2015 appear not to be driven by larger risk premia, and thus may reflect the impact of monetary policy measures adopted by the ECB in order to counteract the fall in inflation expectations and preserve price stability. 
Figure 7

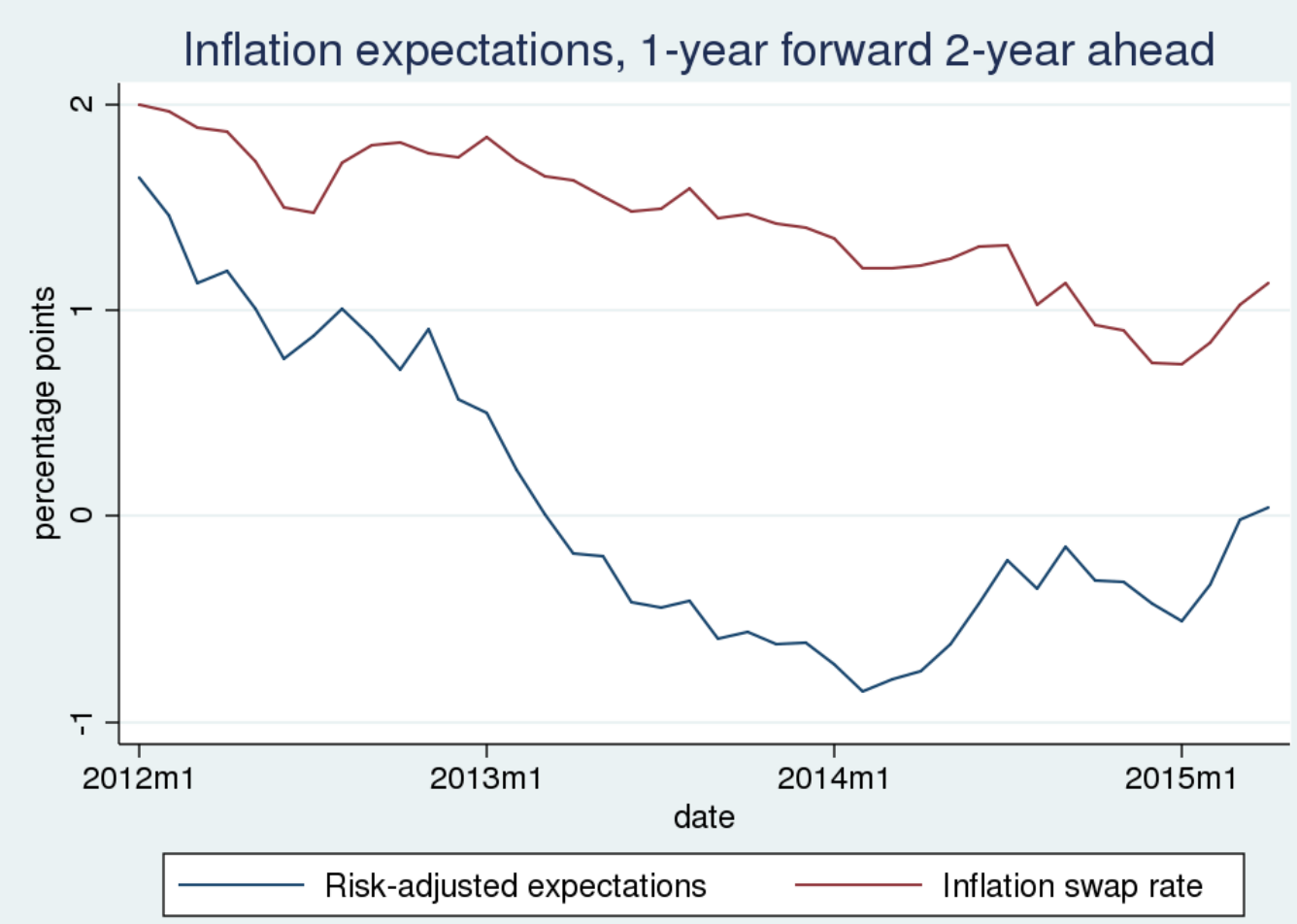

\section{Robustness analysis}

We have performed a number of robustness checks of our main estimates (equation (8)). In our sample the inflation rate abruptly increased in 2008 and then decreased in 2009 , fluctuations linked both to change in the oil prices and the global financial crisis. We therefore estimate our main specification using exclusively data from October 2009 onwards. In this way, we can check whether our results stem from the empirical evidence associated with a very peculiar and turbulent period such as the beginning of the global financial crisis. The estimates are reported in table 4: not only the estimated coefficients are similar to our baseline estimate both in terms of magnitude and statistical significance, but also the $\mathrm{R}^{2}$ of the estimates improves substantially. 
Table 4: equation (9) estimates, sample 2009:10-2015:4

\begin{tabular}{|c|c|c|c|c|c|}
\hline & $e r_{t}^{(1)}$ & $e r_{t}^{(2)}$ & $e r_{t}^{(3)}$ & $e r_{t}^{(4)}$ & $e r_{t}^{(5)}$ \\
\hline Pmi & $\begin{array}{c}-0.0573 * * * \\
(0.0134)\end{array}$ & $\begin{array}{c}-0.0668 * * * \\
(0.0119)\end{array}$ & $\begin{array}{c}-0.0625 * * * \\
(0.0132)\end{array}$ & $\begin{array}{l}-0.00146 \\
(0.0190)\end{array}$ & $\begin{array}{l}-0.0215 \\
(0.0184)\end{array}$ \\
\hline Unemployment & $\begin{array}{c}0.619 * * * \\
(0.0914)\end{array}$ & $\begin{array}{c}1.010 * * * \\
(0.0681)\end{array}$ & $\begin{array}{c}0.975 * * * \\
(0.155)\end{array}$ & $\begin{array}{c}0.791^{* * *} \\
(0.244)\end{array}$ & $\begin{array}{c}0.273 \\
(0.222)\end{array}$ \\
\hline OIS $3 m$ & $\begin{array}{c}0.594 \\
(0.378)\end{array}$ & $\begin{array}{c}1.271^{* * *} \\
(0.0763)\end{array}$ & $\begin{array}{c}0.943 * * * \\
(0.119)\end{array}$ & $\begin{array}{c}0.700 * * * \\
(0.160)\end{array}$ & $\begin{array}{c}0.196 \\
(0.161)\end{array}$ \\
\hline Bid-ask spread & $\begin{array}{l}-2.208^{*} \\
(1.106)\end{array}$ & $\begin{array}{c}0.454 \\
(1.236)\end{array}$ & $\begin{array}{l}-2.135 \\
(1.511)\end{array}$ & $\begin{array}{l}6.149 * \\
(3.531)\end{array}$ & $\begin{array}{l}-4.430 * \\
(2.365)\end{array}$ \\
\hline Constant & $\begin{array}{c}-3.772 * * * \\
(1.087)\end{array}$ & $\begin{array}{c}-7.949 * * * \\
(1.249)\end{array}$ & $\begin{array}{c}-7.014 * * * \\
(1.972)\end{array}$ & $\begin{array}{c}-8.521 * * * \\
(2.123)\end{array}$ & $\begin{array}{c}-1.194 \\
(1.499)\end{array}$ \\
\hline $\begin{array}{l}\text { Observations } \\
\text { R-squared }\end{array}$ & $\begin{array}{c}67 \\
0.672\end{array}$ & $\begin{array}{c}67 \\
0.901\end{array}$ & $\begin{array}{c}67 \\
0.763\end{array}$ & $\begin{array}{c}67 \\
0.562\end{array}$ & $\begin{array}{c}67 \\
0.142\end{array}$ \\
\hline
\end{tabular}

We also re-estimated our model allowing for more lags in the estimation of the covariance matrix. Table 5 reports the estimates when allowing for 10 lag periods. The statistical significance of all the coefficient in the relevant time horizons (i.e. up to 3 years) is unaffected. Using longer lags does not alter statistical significance

Table 5: equation (9) estimates, allowing for 10 lags in variance estimate

\begin{tabular}{lccccc}
\hline & $\mathrm{er}_{t}^{(1)}$ & $\mathrm{er}_{t}^{(2)}$ & $\mathrm{er}_{t}^{(3)}$ & $\mathrm{er}_{t}^{(4)}$ & $\mathrm{er}_{t}^{(5)}$ \\
\hline \multirow{3}{*}{ Pmi } & & & & & \\
& $-0.0845^{* * *}$ & $-0.112^{* * *}$ & $-0.0559^{* * *}$ & 0.00566 & -0.0145 \\
Unemployment & $(0.0223)$ & $(0.0249)$ & $(0.0206)$ & $(0.0164)$ & $(0.0193)$ \\
& $0.800^{* * *}$ & $0.716^{* * *}$ & $0.788^{* * *}$ & 0.285 & 0.268 \\
OIS 3m & $(0.131)$ & $(0.138)$ & $(0.236)$ & $(0.294)$ & $(0.269)$ \\
& $0.781^{* * *}$ & $0.840^{* * *}$ & $0.782^{* * *}$ & 0.321 & 0.206 \\
Bid-ask spread & $(0.229)$ & $(0.141)$ & $(0.148)$ & $(0.211)$ & $(0.212)$ \\
& $1.805^{* * *}$ & -3.713 & -2.104 & -0.318 & -1.591 \\
Constant & $(0.678)$ & $(2.308)$ & $(2.088)$ & $(2.717)$ & $(1.598)$ \\
& $-4.905^{* * *}$ & -2.039 & $-5.511^{* *}$ & -3.310 & -1.687 \\
& $(1.245)$ & $(2.654)$ & $(2.435)$ & $(2.711)$ & $(1.887)$ \\
Observations & 120 & 108 & & & \\
R-squared & 0.360 & 0.682 & 0.512 & 0.380 & 0.121 \\
& & & & & \\
\hline
\end{tabular}

Also among the robustness checks that we do not report here: i) we re-estimated the model sampling the inflation swap rates at the last end of the month, instead of averaging across the month; ii) we substituted longer maturity OIS contract rates for the 3-month rate when estimating longer term inflation swap rates; iii) we used a different indicator of cyclical condition, namely the month on month growth of the industrial production index: in all three checks results remained broadly unchanged. 


\section{Conclusion}

This paper has proposed and adopted an approach to derive risk-adjusted expectations of inflation from inflation-linked securities. In particular, using excess returns of inflation swap rates, we have showed how these could be well predicted by a series of macro-variables available to investors at the time of the inflation swap contract.

We show that since 2012 risk-adjusted measures of inflation expectations seem to have been lower than what implied by swap contracts or, in other words, that risk premia have been positive. More importantly, the decrease in inflation swap rates observed in the second half of 2014 seem to be due to a decrease in inflation expectation rather than a decrease in the inflation risk premia. Finally, also the increase in inflation swap rates observed in the initial months of 2015 is shown that can be explained by an increase in inflation expectations, probably due to the Expanded Asset Purchase Programme undertaken by the ECB, rather than by changes in inflation risk premia. 


\section{Bibliography}

Andreasen, M., "An estimated DSGE model: explaining variation in nominal term premia, real term premia, and inflation risk premia", European Economic Review, 56, 2012.

Cecchetti S., Natoli F. and Sigalotti L., "Tail comovement in option-implied inflation expectations as an indicator of anchoring", Banca d'Italia working paper, Forthcoming, 2015

Cochrane. J. H. and Piazzesi, M., "Bond Risk Premia", American Economic Review, 95(1), 2005.

Fleckenstein, M., Longstaff F. and Lusting, H., "Deflation risk", NBER Working Paper 19238, 2013.

Haubrich, J., Pennacchi, G., and Ritchken, P., "Inflation expectations, real rates and risk premia: evidence from inflation swaps", The Review of Financial Studies, 25, 2012.

Hördahl, P. and Tristani, O., "Inflation risk premia in the term structure of interest rates", Journal of the European Economic Association, 10(3), 2012.

Hördahl, P. and Tristani, O., "Inflation risk premia in the euro area and the United States", International Journal of Central Banking, 10(3), 2014.

Pericoli, M., "Expected inflation and inflation risk premium in the euro area and in the United States", Banca d'Italia working paper, no. 842, 2012.

Piazzesi, M. and Swanson, E. T., "Futures prices as risk-adjusted forecasts of monetary policy", Journal of Monetary Economics, 55, 2008. 